\title{
Type Ic core-collapse supernovae evolved from very massive stars
}

\section{Takashi Yoshida*}

Yukawa Institute for Theoretical Physics, Kyoto University, Kyoto 606-8502, Japan

E-mail: voshidadyukawa.kyoto-u.ac.ip

\section{Shinpei Okita}

Department of Astronomy, Graduate School of Science, University of Tokyo, Tokyo 113-0033, Japan

\section{Hideyuki Umeda}

Department of Astronomy, Graduate School of Science, University of Tokyo, Tokyo 113-0033, Japan

E-mail: umedadastron.s.u-tokvo.ac.jp

We investigate the possibility of Type Ic super-luminous core-collapse supernovae producing large amounts of ${ }^{56} \mathrm{Ni}$. We calculate aspherical energetic explosions of core-collapse supernovae evolved from very massive stars. We then calculate the explosive nucleosynthesis and evaluate the total ejecta mass and the velocity distributions of the ejecta. The adopted supernova progenitors are 43.2 and $61.1 M_{\odot}$ WO stars evolved from 110 and $250 M_{\odot}$ stars with the metallicity $Z=0.004$. The aspherical core-collapse supernovae produce $3.7-6.1 M_{\odot}{ }^{56} \mathrm{Ni}$ depending on the stellar mass and asphericity. The ejected ${ }^{56} \mathrm{Ni}$ yield reproduces the yield ejected from the observations of SN 2007bi. These models also indicate large velocity dispersions in the ejecta masses of $\mathrm{Fe}, \mathrm{Ca}, \mathrm{Si}$, and $\mathrm{O}$. Fast $\mathrm{Fe}$ and $\mathrm{Ca}$ components and slow $\mathrm{O}$ component shown in the aspherical explosion models are consistent with the observations of SN 2007bi. An aspherical core-collapse supernova evolved from a very massive star is a possibility of the explosion of SN 2007bi.

XIII Nuclei in the Cosmos,

7-11 July, 2014

Debrecen, Hungary

\footnotetext{
*Speaker.
} 


\section{Introduction}

Super-luminous supernovae ( $\mathrm{SNe}$ ) have been found as very bright $\mathrm{SNe}$ with the maximum absolute magnitude smaller than -21 in distant galaxies [四. These SNe belong to Type IIn or Type Ic and have a variety in the decline time scale of their light curves []]. Their explosion mechanism have not been clarified. Several possibilities were proposed such as the interaction with surrounding circumstellar material (e.g., [B] ]), spinning-down nascent magnetar [四], pair-instability SNe []], and energetic core-collapse $\mathrm{SNe}$ [目].

SN 2007bi is a Type Ic super-luminous SN [0]. This SN indicated slow decline of the light curve and the decline time scale was well fitted by the radioactive decay of ${ }^{56} \mathrm{Co}$. The ${ }^{56} \mathrm{Ni}$ amount in the SN ejecta was evaluated from the analyses of the light curve and spectra. The evaluated ${ }^{56} \mathrm{Ni}$ amount is $3.5-7.4 M_{\odot}$ and is much larger than those evaluated from observed hypernovae. The host galaxy of SN 2007bi was identified and the metallicity was measured to be $Z=0.2-0.4 Z_{\odot}$ [四]. In order to explain the large ${ }^{56} \mathrm{Ni}$ amount, a pair-instability $\mathrm{SN}$ with a $95-105 M_{\odot} \mathrm{CO}$ star progenitor was proposed [5]. On the other hand, an energetic core-collapse $\mathrm{SN}$ of a $\sim 40 M_{\odot} \mathrm{CO}$ star was also proposed as the explosion mechanism [焑]. Some energetic Type Ic SNe indicated observational evidence for aspherical explosions in late-time spectra [ 8$]$. Hypernovae as aspherical energetic core-collapse SNe have been discussed as an origin of extremely metal-poor stars [Q]. Therefore, aspherical explosion is also possible for energetic core-collapse SNe. An aspherical energetic SN explosion has a possibility for ejecting a large ${ }^{56} \mathrm{Ni}$ amount enough to reproduce the observations of SN 2007bi.

In this study, we investigate the possibility of aspherical core-collapse explosion of SN 2007bi. We concentrate on the discussion about the ${ }^{56} \mathrm{Ni}$ amount ejected from aspherical $\mathrm{SN}$ explosions and the velocity distributions of the $\mathrm{Fe}, \mathrm{Ca}, \mathrm{Si}$, and $\mathrm{O}$ ejecta. Detail for this study is discussed in [ए0].

\section{Very massive star and core-collapse SN explosion models}

We used very massive star models with the initial masses of 110 and $250 M_{\odot}$ for the calculations of core-collapse $\mathrm{SNe}[\mathbf{W}]$. The metallicity of the stars is set to be $Z=0.004\left(0.2 Z_{\odot}\right)$, corresponding to the metallicity of the host galaxy of SN 2007bi. We calculated the evolution of these stars from hydrogen burning to the onset of the core-collapse. These stars lost all of the $\mathrm{H}$ rich envelope and He layer during the evolution. They finally evolved to 43.1 and $61.1 M_{\odot}$ WO stars. The $250 M_{\odot}$ star experienced pulsational pair-instability during $\mathrm{O} / \mathrm{Si}$ burning.

We calculated aspherical energetic SN explosions of these stars using 2D hydrodynamical code [U]. We first prepared the collapsed progenitor of which central density is about $10^{14} \mathrm{~g} \mathrm{~cm}^{-3}$ in order to mimic the collapse to form a black hole as a remnant. Then, we injected the kinetic energy within the area of an opening angle $\theta_{\text {op }}$ at the location of the mass cut, i.e., $2.0 M_{\odot}$ in the mass coordinate. The injected kinetic energy is $5 \times 10^{52}$ and $7 \times 10^{52}$ ergs for 110 and $250 M_{\odot}$ models, respectively. We pursued the $\mathrm{SN}$ explosions with six cases of the opening angles, i.e., $\theta_{\mathrm{op}}=8^{\circ}, 11.25^{\circ}, 22.5^{\circ}, 45^{\circ}, 67.5^{\circ}$, and $90^{\circ}$ (spherical explosion). We followed the motions of 5,200 tracer particles during the explosions. Then, we calculated the explosive nucleosynthesis during the SN explosion by postprocessing using the thermal evolution of the tracer particles. The nuclear reaction network in this study consists of 288 species of nuclei [प]]. 


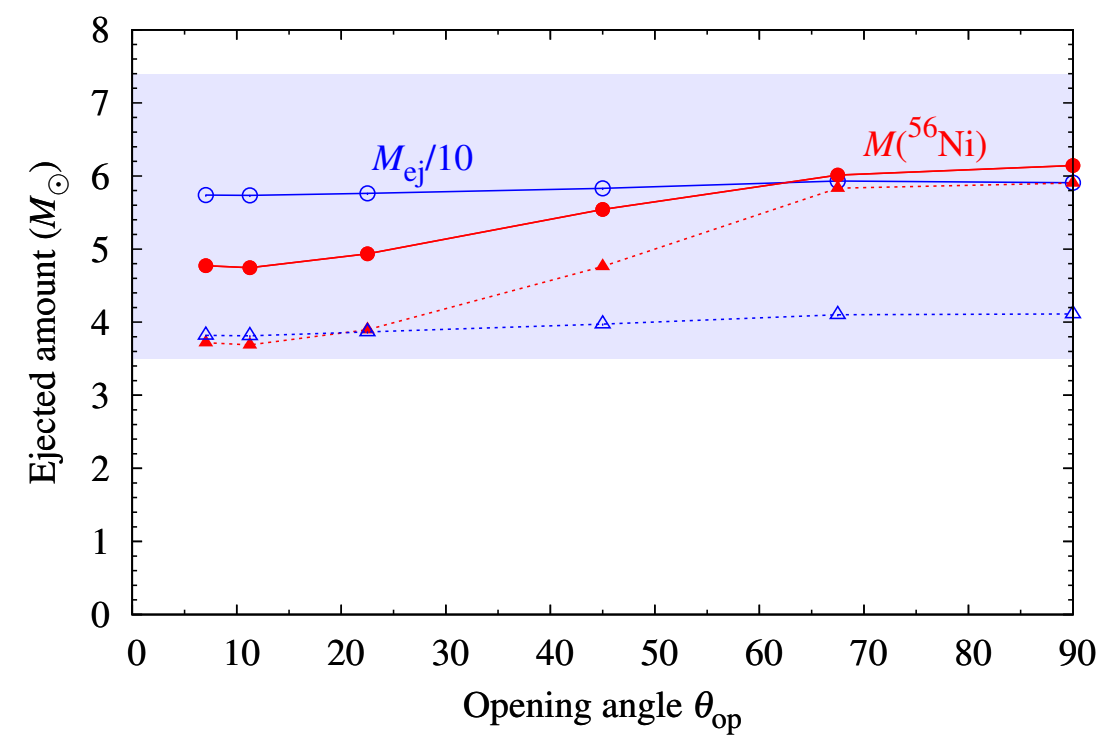

Figure 1: The ejected ${ }^{56} \mathrm{Ni}$ amount (red lines with close symbols) and the total ejecta mass divided by 10 (blue lines with open symbols) as a function of the opening angle $\theta_{\text {op }}$ in the $250 M_{\odot}$ model (solid lines with circles) and the $110 M_{\odot}$ model (dashed lines with triangles). Blue-shaded region indicates the range of the ${ }^{56} \mathrm{Ni}$ amount observed in SN 2007bi.

\section{3. ${ }^{56} \mathrm{Ni}$ amount in aspherical $\mathrm{SNe}$}

We show the dependence of the ejected ${ }^{56} \mathrm{Ni}$ amount $\left(M\left({ }^{56} \mathrm{Ni}\right)\right)$ and the ejecta mass $\left(M_{\mathrm{ej}}\right)$ on the opening angle for the 250 and $110 M_{\odot}$ models in Fig. 1 . In both models, the ejected ${ }^{56} \mathrm{Ni}$ amount increases with the opening angle. The ${ }^{56} \mathrm{Ni}$ amount is in the range of $4.8-6.1$ and $3.7-5.9 M_{\odot}$ for the 250 and $110 M_{\odot}$ models, respectively. We also see the increase in the total ejecta mass with the opening angle but the increased steepness is less than that of the ${ }^{56} \mathrm{Ni}$ amount. The dependence of the ${ }^{56} \mathrm{Ni}$ amount is mainly due to the fallback of the innermost region. ${ }^{56} \mathrm{Ni}$ is synthesized through the explosive nucleosynthesis in the innermost region of the ejecta. The synthesized ${ }^{56} \mathrm{Ni}$ amount scarcely depends on the opening angle. However, in small opening angle models, some materials on the equatorial plane in the innermost region fall onto the central remnant. The fallen amount is larger for smaller opening angle models. The main component of the fallen material is ${ }^{56} \mathrm{Ni}$. Since the main component of the innermost region is ${ }^{56} \mathrm{Ni}$, the dependence of the ${ }^{56} \mathrm{Ni}$ amount on the opening angle is larger than that of the total ejecta mass.

We compare the ejected ${ }^{56} \mathrm{Ni}$ amount of the 250 and $110 M_{\odot}$ models with the ${ }^{56} \mathrm{Ni}$ amount evaluated from the observations of SN $2007 \mathrm{bi}\left(3.5 \leq M\left({ }^{56} \mathrm{Ni}\right) \leq 7.4 M_{\odot}\right.$; see blue-shaded region in Fig. 1). Although the calculated ${ }^{56} \mathrm{Ni}$ amounts of these models depend on the opening angle, they are within the range of the ${ }^{56} \mathrm{Ni}$ amount observed in SN 2007bi. Therefore, aspherical core-collapse $\mathrm{SN}$ explosion evolved from a $\sim 110-250 M_{\odot}$ star would reproduce ${ }^{56} \mathrm{Ni}$ amount ejected from $\mathrm{SN}$ $2007 \mathrm{bi}$. 

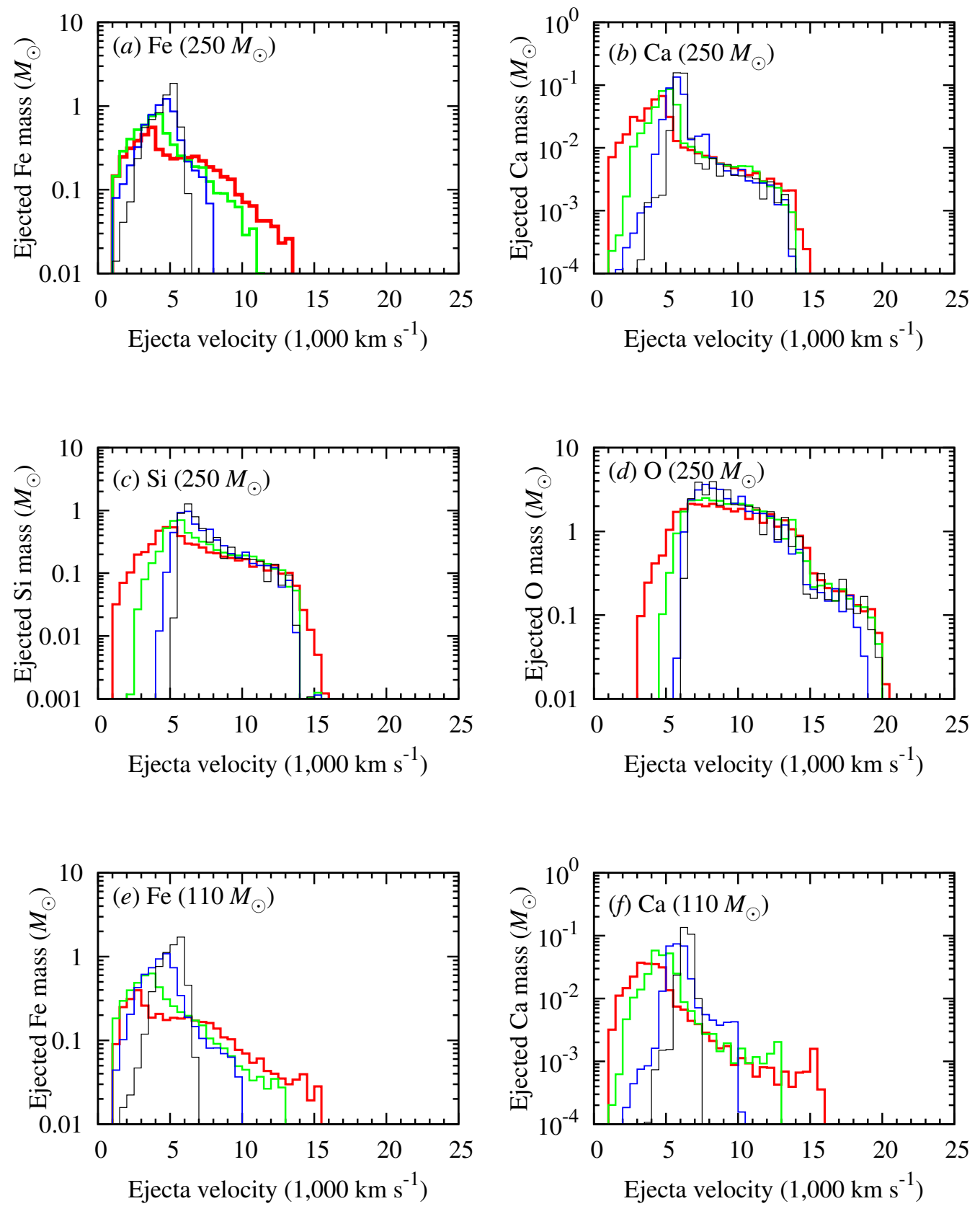

Figure 2: The dependence of ejected elemental mass on the ejecta velocity. Panels $(a)-(d)$ indicate the ejected masses of $\mathrm{Fe}, \mathrm{Ca}, \mathrm{Si}$, and $\mathrm{O}$ in the $250 M_{\odot}$ model. Panels $(e)$ and $(f)$ indicate the ejected $\mathrm{Fe}$ and $\mathrm{Ca}$ masses in the $110 M_{\odot}$ model, respectively. The red, green, blue, and black lines correspond to the cases of the opening angles $\theta_{\mathrm{op}}=11.25^{\circ}, 45^{\circ}, 67.5^{\circ}$, and $90^{\circ}$, respectively. 


\section{Velocity distribution of aspherical SN ejecta}

The velocity dispersions of $\mathrm{Fe}, \mathrm{Ca}, \mathrm{Si}$, and $\mathrm{O}$ in the ejecta of SN 2007bi have been analyzed from its spectra $[\square]$. Here, we evaluate the velocity distributions of $\mathrm{Fe}, \mathrm{Ca}, \mathrm{Si}$, and $\mathrm{O}$ in the aspherical SN explosions of the 250 and $110 M_{\odot}$ models. Panels $(a)-(d)$ of Fig. 2 show the velocity distributions of these elements in the $250 M_{\odot}$ model. The velocity dispersion of each element increases with decreasing in the opening angle. The Fe velocity is in the range of $1,000-6,500 \mathrm{~km}$ $\mathrm{s}^{-1}$ in the spherical explosion. On the other hand, it exceeds $10,000 \mathrm{~km} \mathrm{~s}^{-1}$ in aspherical explosions with $\theta_{\mathrm{op}}=45^{\circ}$. The maximum Fe velocity is $13,500 \mathrm{~km} \mathrm{~s}^{-1}$ in the aspherical explosion with $\theta_{\mathrm{op}}=11.25^{\circ}$. Fast component of the Ca ejecta scarcely depends on the opening angle. The maximum velocities of the $\mathrm{SN}$ models are $14,000-15,000 \mathrm{~km} \mathrm{~s}^{-1}$. Although $\mathrm{Fe}$ and $\mathrm{Ca}$ are mainly produced in the innermost region, aspherical explosions enable to eject these elements with faster velocity from the polar axis.

The $\mathrm{O}$ velocity is in the range of $5,500-19,000 \mathrm{~km} \mathrm{~s}^{-1}$ in the spherical explosion. On the other hand, the minimum $O$ velocity is $3,000 \mathrm{~km} \mathrm{~s}^{-1}$ in the aspherical explosion with $\theta_{\mathrm{op}}=11.25^{\circ}$. The fallback on the equatorial plane reduces the velocity of O-rich ejecta in aspherical explosions.

Panels $(e)$ and $(f)$ of Fig. 2 show the velocity distributions of $\mathrm{Fe}$ and $\mathrm{Ca}$ in the $110 M_{\odot}$ models, respectively. The dependence of the velocity dispersions on the opening angle in the 110 $M_{\odot}$ model is similar to the $250 M_{\odot}$ model. The maximum velocities of $\mathrm{Fe}$ and $\mathrm{Ca}$ are $15,500 \mathrm{~km}$ $\mathrm{s}^{-1}$ and $16,000 \mathrm{~km} \mathrm{~s}^{-1}$, respectively. These velocities are faster than the corresponding ones in the $250 M_{\odot}$ models.

Photospheric velocities of $\mathrm{Fe}, \mathrm{Ca}, \mathrm{Si}$, and $\mathrm{O}$ were analyzed in SN 2007bi [ $[\mathrm{d}]$. The velocities of the Fe II $\lambda 5037$ were measured to be $\sim 12,000 \mathrm{~km} \mathrm{~s}^{-1}$. The Ca velocities are 12,000-16,000 $\mathrm{km} \mathrm{s}^{-1}$ for the Ca H\&K $\lambda 3976$ and also indicated slower velocities of $\sim 8,000-10,000 \mathrm{~km} \mathrm{~s}^{-1}$ for the Ca II near-IR $\lambda 8571$. The velocities of the Si II $\lambda 6355$ are in the range of $\sim 5,500-8,400$ $\mathrm{km} \mathrm{s}^{-1}$. Lower limit of the velocities of the O I $\lambda 7774$ was measured to be $4,500 \mathrm{~km} \mathrm{~s}^{-1}$.

The very high velocities of the Fe and Ca expansion were obtained in SN 2007bi. In order to reproduce such high velocities, the explosion should be aspherical or large scale mixing should have occurred in spherical explosion. In our study, the high expansion velocities observed in the $\mathrm{Fe}$ and $\mathrm{Ca}$ lines of SN 2007bi are shown in the aspherical explosion models with the opening angle $\theta_{\mathrm{op}} \leq 45^{\circ}$. The slow component of the $\mathrm{O}$ velocities are also obtained in the aspherical explosion models. Thus, the explosion velocities of aspherical explosion models evolved from very massive stars with the initial masses of $\sim 110-250 M_{\odot}$ are consistent with photospheric velocities observed in SN 2007bi.

\section{Conclusions}

We investigated a possibility of Type Ic super-luminous $\mathrm{SNe}$ as aspherical core-collapse $\mathrm{SN}$ explosions evolved from very massive stars. We pursued 2D hydrodynamical simulations of aspherical SN explosions with different opening angles for the 110 and $250 M_{\odot}$ star models. Then, we followed the explosive nucleosynthesis of these SNe by postprocessing. The ${ }^{56} \mathrm{Ni}$ amount of the aspherical SNe increases with the opening angle of the models. The range of the ${ }^{56} \mathrm{Ni}$ amount is $3.7-6.1 M_{\odot}$. The obtained range is consistent with the amount observed in SN 2007bi. We also 
investigated the asphericity dependence of the velocity distributions of $\mathrm{Fe}, \mathrm{Ca}, \mathrm{Si}$, and $\mathrm{O}$ in the $\mathrm{SN}$ ejecta. Fast components of the $\mathrm{Fe}$ and $\mathrm{Ca}$ ejecta achieve to $\sim 13,000-15,000 \mathrm{~km} \mathrm{~s}^{-1}$ in aspherical explosions. Fast components of the $\mathrm{Fe}$ and $\mathrm{Ca}$ ejecta and slow component of $\mathrm{O}$ ejecta obtained in this study are also observed in the expansion velocities in SN 2007bi. These observational features indicate that an aspherical core-collapse SN evolved from a very massive star is a possibility of Type Ic super-luminous SN 2007bi.

This work was supported by the Grants-in-Aid for Scientific Research (20041005, 20105004, 23540287, 24244028, 26400271).

\section{References}

[1] R.M. Quimby, et al. Nature 474 (2011) 487.

[2] A. Gal-Yam, Science 337 (2012) 927.

[3] R.A. Chevalier, C.M. Irwin, Astrophys. J. Lett. 729 (2011) L6.

[4] S.E. Woosley, Astrophys. J. Lett. 719 (2010) L204.

[5] A. Gal-Yam, et al., Nature 462 (2009) 624.

[6] T. Moriya, N. Tominaga, M. Tanaka, K. Maeda, K. Nomoto, Astrophys. J. 717 (2010) L83.

[7] D.R. Young, et al., Astron. Astrophys 512 (2010) A70.

[8] K. Maeda, et al., Science 319 (2008) 1220.

[9] N. Tominaga, Astrophys. J. 690 (2009) 526.

[10] T. Yoshida, S. Okita, H. Umeda, Mon. Not. R. Astron. Soc. 438 (2014) 3119.

[11] S. Okita, H. Umeda, T. Yoshida, in Origin of Matter and Evolution of Galaxies 2011, AIP Conf. Ser. 1484 (2012) 418.

[12] T. Yoshida, H. Umeda, Mon. Not. R. Astron. Soc. 412 (2011) L78. 